\title{
THE BOUNDARY OF THE NUMERICAL RANGE
}

\author{
by G. DE BARRA
}

(Received 19 July, 1979)

1. Introduction. In [1] it was shown that for a compact normal operator on a Hilbert space the numerical range was the convex hull of the point spectrum. Here it is shown that the same holds for a semi-normal operator whose point spectrum satisfies a density condition (Theorem 1 ). In Theorem 2 a similar condition is shown to imply that the numerical range of a semi-normal operator is closed. Some examples are given to indicate that the condition in Theorem 1 cannot be relaxed too much.

2. The bounded operator $T$ on a Hilbert space $H$ is hyponormal if $T^{*} T \geqq T T^{*}$ and semi-normal if either $T$ or $T^{*}$ is hyponormal. A useful reference is [7]. Some properties are now given.

Lemma 1. Let $T$ be semi-normal on $H$. Then $\overline{W(T)}=$ coSp $T$, where $W(T)$ denotes the numerical range of $T, \mathrm{Sp} T$ denotes the spectrum of $T$ and co denotes convex hull. trivial.

This is due to Putnam [6] and Stampfli [8] for hyponormal operators; the extension is

Lemma 2. Let $T$ be semi-normal on $H$. Then the extreme points of $W(T)$ lie in the point spectrum of $T$.

Proof. For normal operators this is due to MacCluer [4] and for hyponormal operators to Stampfli [9]. So suppose $T^{*}$ is hyponormal. Write ex $A$ for the extreme points of $A$ and $\operatorname{pSp} T$ for the point spectrum of $T$. Then $z \in \operatorname{ex} W(T)$ implies $z^{*} \in$ ex $W\left(T^{*}\right)$; but then $z^{*} \in \operatorname{pSp} T^{*}$ and so $z \in \operatorname{pSp} T([\mathbf{8}$, Lemma 2]).

Lemma 3. Let $T$ be a hyponormal operator, $L$ a support line for $W(T)$ and

$$
N=\bigcup_{z \in L}\left\{x:(T x, x)=z\|x\|^{2}\right\} .
$$

Then $N$ is a reducing subspace for $T$ and $T \mid N$ is normal.

Proof. It is shown in [2, Lemma 2] that $N$ is a subspace and that

$$
N=\left\{x: e^{-i \theta}(T-z I) x=e^{i \theta}\left(T^{*}-z^{*} I\right) x\right\}
$$

for all $z \in L$, where $\theta$ is the acute angle between $L$ and the $x$-axis. So if $z$ is any fixed point on $L$ and $S=T-z I$,

$$
N=\left\{x: S x=e^{2 i \theta} S^{*} x\right\} .
$$

But as $S$ is hyponormal, Lemma 3 of [9] gives that $N$ reduces $S$ and $S \mid N$ is normal. So the same holds for $T$. That, for any operator, such a subspace $N$ is reducing whenever it is invariant is observed in [3] (page 406).

Glasgow Math. J. 22 (1981) 69-72. 


\section{The main results}

THEOREM 1. Let $T$ be a semi-normal operator and assume $\mathrm{pSp} T \cap C$ dense in

$$
C=\operatorname{Sp} T \cap \partial(\operatorname{cosp} T) \text {. }
$$

Then $W(T)=\operatorname{co}(\mathrm{pSp} T)$.

Proof. We suppose first that $T$ is hyponormal. For $z \in W(T)$ we wish to show $z \in \operatorname{co}(\mathrm{pSp} T)$ and we may suppose $z=0$.

(i) Suppose $0 \in \partial W(T)$, the boundary of the numerical range, and that im $W(T) \leqq 0$. Let $L$ be the horizontal support line at 0 . Let $N$ be as in Lemma 3 so that $T_{0}=T \mid N$ is Hermitian. Write $L_{0}=L \cap W(T)$. If $L_{0}=\{0\}$ or 0 is an end-point of $L_{0}$, then $0 \in \operatorname{ex} W(T)$ by Lemma 2 . Suppose $L_{0}$ is the interval $(\alpha, \beta)$ where $\alpha<0<\beta$; if either end-point is in $L_{0}$ it is in pSp $T$ and the argument is shortened. Then $\operatorname{Sp} T_{0} \cap(\alpha, 0)$ and $\operatorname{Sp} T_{0} \cap(0, \beta)$ meet pSp $T_{0}$. For $\alpha$ and $\beta$ are not isolated points of $\mathrm{pSp} T_{0}$, so $\operatorname{Sp} T_{0} \cap(\alpha, 0)$ and $\operatorname{Sp} T_{0} \cap(0, \beta)$ are non-empty. Also we can find $z, z^{\prime}, y \in \operatorname{Sp} T_{0}$ with $\alpha<z^{\prime}<z<0<y<\beta$. Let $M$ be a circular neighbourhood of $z$ not meeting $z^{\prime}$ or 0 . If $M$ meets $\partial(\operatorname{cosp} T)$ in the lower half-plane, at $z^{\prime \prime}$ say, choose a neighbourhood $M_{0}$ of $z$ within the triangle $z^{\prime}, z^{\prime \prime}, y$, with $M_{0} \subset M$ so that $M_{0} \cap C \subset L_{0}$. Then by the density hypothesis pSp $T$ meets $M_{0} \cap C$. Similarly it meets $(0, \beta)$, so $0 \in \operatorname{co}(\mathrm{pSp} T)$.

(ii) Now suppose $0 \in$ int $W(T)$ and that a square centered at 0 with a corner at $(4 d, 4 i d)$ lies in $W(T)$. If $A=(2 d, 2 i d), A \in \overline{c o}(\operatorname{ex}(\operatorname{cosp} T))$. So choose $A^{\prime}$ in the same quadrant with $\left|A-A^{\prime}\right|<d$ and $A^{\prime} \in \operatorname{co}(\operatorname{ex}(\cos S T))$, say

$$
A^{\prime}=\sum_{i=1}^{n} \lambda_{i} z_{i}
$$

where $z_{i} \in \operatorname{Sp} T \cap \partial(\operatorname{coSp} T), \lambda_{i}$ are non-negative and $\sum_{i=1}^{n} \lambda_{i}=1$. Let $z_{i}^{\prime} \in \mathrm{pSp} T$ with $\left|z_{i}-z_{i}^{\prime}\right|<d / n$. So $A^{\prime \prime}=\sum \lambda_{i} z_{i}^{\prime}$ lies in co(pSp $\left.T\right)$ and $A^{\prime \prime}$ is in the first quadrant. Similarly for the other quadrants. So $0 \in \operatorname{co}(\mathrm{pSp} T)$.

Now suppose that $T^{*}$ is hyponormal. Suppose $L^{*}$ is a support line for $W\left(T^{*}\right)$ and $N^{*}$ is the reducing subspace for $T^{*}$ provided by Lemma 3. Then $L$ supports $W(T)$. Also $N^{*}$ reduces $T$; indeed $N^{*}$ is just the subspace $N$ since $(T x, x)=z\|x\|^{2}$ whenever $\left(T^{*} x, x\right)^{*}=$ $z\|x\|^{2}$. Since $T \mid N$ is normal, the proof in (i) proceeds as before. For (ii) we only need the convexoid property provided by Lemma 1 for $T^{*}$ and hence for $T$.

Corollary. If $T$ is hyponormal and satisfies the conditions of the theorem then $W\left(T^{*}\right)=\operatorname{co}\left(\mathrm{pSp} T^{*}\right)$.

Proof. Since $(\mathrm{pSp} T)^{*} \subseteq \mathrm{pSp} T^{*}$, we have $W\left(T^{*}\right) \subset \operatorname{co}\left(\mathrm{pSp} T^{*}\right)$ and the result follows.

The following theorem is of the same kind. The spectral case when $T$ is normal was proved by Meng [5] by a direct method.

Theorem 2. Let $T$ be semi-normal. Then a necessary and sufficient condition that $W(T)$ be closed is that $\operatorname{ex}(\operatorname{cosp} T)$ lie in $\mathrm{pSp} T$. 
Proof. The condition implies co Sp $T \subseteq \operatorname{co}(\mathrm{pSp} T)$ so $\overline{W(T)} \subseteq W(T)$, using Lemma 1 , so the condition is sufficient. Also the condition is necessary for, if $W(T)$ is closed, ex $W(T)=\operatorname{ex}(\operatorname{cosp} T)$. But ex $W(T) \subseteq \mathrm{pSp} T$ (Lemma 2).

Some examples are now given to illustrate the conditions.

(i) Let $T$ be the compact diagonal operator with diagonal elements

$$
1, i, \frac{1+i}{2}, \frac{1+i}{3}, \frac{1+i}{4}, \ldots
$$

Here the set $C$ of Theorem 1 consists of $1, i, 0$ so the condition of Theorem 1 is not satisfied but the conclusion holds. This case is covered by the theorem of [1] referred to in $\S 1$. Here $W(T)$ is not closed. Note that for compact operators normality and seminormality are equivalent.

(ii) Let $T_{1}$ be the diagonal operator with diagonal elements

$$
1,1 / 2,1 / 3, \ldots,
$$

let $T_{2}=T_{1}+i I$ and let $T=T_{1} \oplus T_{2}$. Here the set $C$ is $\mathrm{Sp} T$. Also $W(T)$ is the square having corners $0,1,1+i$, without the side $[0, i]$. Theorem 1 applies, Theorem 2 does not.

(iii) The following example shows that $\mathrm{pSp} T$ dense in $\partial \mathrm{Sp} T$ is not sufficient for Theorem 1. Let $T_{1}$ be a diagonal operator with diagonal entries on the lines

$$
L_{n}=\left[\frac{1}{n}, \frac{1}{n}+i\right], \quad n=1,2, \ldots
$$

and including the end-points. Let $T_{2}$ be Hermitian with $W\left(T_{2}\right)=(0,1)$ and let $T=$ $T_{1} \oplus i T_{2}$. Then $W(T)$ is the square having corners $0,1,1+i, i$ but with the corners $0, i$ removed. So $W(T) \neq \operatorname{co}(\mathrm{pSp} T)$ but $\mathrm{pSp} T$ is dense in $\partial \mathrm{Sp} T=\operatorname{Sp} T$.

I would like to acknowledge the helpful suggestions of Mary Embry-Wardrop.

\section{REFERENCES}

1. G. de Barra, J. R. Giles and B. Sims, On the numerical range of operators, J. London Math. Soc. (2) 5 (1972), 704-706.

2. M. R. Embry, The numerical range of an operator, Pacific J. Math. 32 (1970), 647-650.

3. M. R. Embry, Orthogonality and the numerical range, J. Math. Soc. Japan 27 (3) (1975), 405-411.

4. C. R. MacCluer, On extreme points of the numerical range of normal operators, Proc. Amer. Math. Soc. 16 (1965), 1183-1184.

5. Ching-Hwa Meng, A condition that a normal operator have a closed numerical range, Proc. Amer. Math. Soc. 8 (1957), 85-88.

6. C. R. Putnam, On the spectra of semi-normal operators, Trans. Amer. Math. Soc. 119 (1965), 509-523.

7. J. G. Stampfii, Hyponormal operators, Pacific J. Math. 12 (1962), 1453-1458.

8. J. G. Stampfli, Hyponormal operators and spectral density, Trans. Amer. Math. Soc. 117 (1965), 469-476. 
9. J. G. Stampfli, Extreme points of the numerical range of a hyponormal operator, Michigan Math. J. 13 (1966). 87-89.

Department of Mathematics

Royal Holloway College

EGHAM

SURREY, U.K. 